\title{
Waist-Hip Ratio
}

National Cancer Institute

\section{Source}

National Cancer Institute. Waist-Hip Ratio. NCI Thesaurus. Code C17651.

The ratio of the abdominal circumference at the navel to maximum hip and buttocks circumference; looks at the proportion of fat stored on the body around the waist and hip. Carrying extra weight around the waist is a greater health risk than carrying extra weight around the hips or thighs. 